\title{
STOCK ASSESMENT, BIOMASS AND FISH PRODUCTION IN TWO MEDITERRANEAN BASINS (NE Spain)
}

\author{
L. Zamora', D. Saavedra ${ }^{2}$ and R.Moreno-Amicht ${ }^{1}$ \\ 1 Instituto de Ecologia Acuática y Depto. de Ciencias Ambientales, Facultad de Ciencias, Universidad de Girona. Campus de \\ Montilivi. 17071-Girona \\ 2. Parque Natural de "Aiguamolls de l'Empordà". Mas "El Cortalet". 17486-Caste116 d'Empúries
}

Keywords: Population estimation, production, removal method, electrofishing, Muga, Fluvih. Aiguamolls de l'Empordà, Eurasian otter.

Palabras clave: Estima de población, producción, método de extracción, pesca eléctrica, Muga, Fluviá, Aiguamolls de l'Empordá, nutria eurásica.

\begin{abstract}
In order to evaluate the success of the reintroduction of the Eurasian otter (Lutra lutra) in the Empordh wetlands ("Parc Natural dels Aiguamolls de l'Empordà"), and the Muga and Fluvih basins, density of fish, biomass and production in the Muga and Fluvià Rivers have been estimated, since fishes represent the principal prey in the otter diet. 12 study sites were selected in order to survey the main flows in both basins. Electrofishing surveys were conducted by blocking off the station with barrier nets, which was performed upon 3 successive catches. The density estimated presents a range of 1,136-125 ind 'ha' in the Muga basin, 4,49-163 ind.ha' in the Fluvih basin and 3,76-52,2 ind $\cdot \mathrm{ha}^{-1}$ in the Empordh wetlands. Estimated biomass ranges are 0,616-277,6 g.m ${ }^{2}, 8,79-351,2 \mathrm{~g} \cdot \mathrm{m}^{2}$, and 5,7-108 g. $\mathrm{m}^{-2}$, respectively. These density and biomass ranges are similar to other results obtained from rivers inhabited by the Eurasian otter in NE Spain.
\end{abstract}

\section{INTRODUCTION}

Eurasian otters (Lutra lutra) inhabited the Fluvih and Muga basins and Empordh wetlands untill the middle of this century when, due to different factors, such as hunting, loss of habitat and pollution, its population began to decrease until it became completely extinguished in the 80's (RUIZ-OLMO, 1995; SAAVEDRA \& SARGATAL, 1993).

The staff in "Parc Natural dels Aiguamolls de l'Empordà" has started recently the Otter Project in order to reintroduce the Eurasian otter in the Natural Park and the Fluvih and Muga rivers, whose currents flow into the wetlands, since it presented stable populations in the past and is a well candidate to point to a good status of the habitat where it is

Limnética, 12 (1): 47-58 (1996)

(C) Asociación Española de Limnologia, Madrid. Spain present. The success of a reintroduction has to be assessed. Therefore, this project has been divided into four parts in order to evaluate the success of the reintroduction as: 1) prospection of basins involves for otter's traces, 2) study of habitat state, 3) to establish the level of pollutants at the sediment and fishes, and 4) stock assessment of fish populations as major otter's prey.

To set up the causes ot the disappearance and ensure that these have been suppressed represents the first step in starting a project of this type. After verifying that otters are absent throughout the study area, that the habitat is available and that the level of pollutants is low (SAAVEDRA, 1995) there only remains to assess the population of the principal prey. 
The reintroduction of a species in a system, even when it had been present before, may represent a perturbation of the community structure and dynamics. This disturbance may result in the appearance of non-equilibrium communities characterised by fluctuating populations. extinction, and low predictability of community composition (MEFFE, 1984).

In the case of an introduced species which belonged to a high predator trophic level the perturbation can be expressed in terms of predator-prey interaction and an expected response takes place when some details of this interaction are available. This includes knowledge of the species diversity, relative prey abundance, size structure, individuals fitness (measure of survivorship or fecundity of prey individuals), individuals growth and behaviour (SIH et al., 1985).

The reintroduction of otters will involve a relationship between this predator and the fish community available so it represents the main prey of its annual diet (MASON \& MCDONALD, 1986). This predator-prey interaction will be classified in terms of their "expected" Or "unexpected" response according to the amount of information available. The aim of this work is to estimate population sizes, biomasses and production of fish communities in order to establish a framework to guarantee the success of the otter reintroduction with regard to the principal prey source.

\section{STUDY AREA, MATERIAL AND METHODS}

Studies involved the Muga and Fluvifi drainage basins (Figure I) Both the Muga and thc Fluvià are low headwaters Mediterranean rivers with irregular water regime, fed by surface waters (rainfall) and lower flow tipyfied by a low absolute volume. The Muga basin presents a surface of 853.78 $\mathrm{km}^{2}$ and an average flow of $2,44 \mathrm{~m}^{3} \cdot \mathrm{sg}$. The total length of its main branch is $64,7 \mathrm{~km}$. The Fluvià rivel- is $97,2 \mathrm{~km}$ long, with a mean flow of $1,27 \mathrm{~m}^{3} \cdot \mathrm{sg}$ ' and a basin surface of 1123,58 $\mathrm{km}^{2}$ (BRUSI, 1992). Polution levels are, in general, low. Both rivers flow into "Aiguamolls de I'Empordà", wetlands protected as a natural park.

In November 1995, several sets of data were collected by the Natural Park biologists in succesive catches of fish. 12 sites were selected in order to survey the main flows of both basins. The general characterization of each site was obtained (Table I). Lengths of these sampling sites varied within the range of 50 and 150 meters.
FIGURE 1: Geographic location of the study area showing the Muga and Fluvià basins and Empordà wetland.

FIGURA 1. Localización geográfica del área de estudio mostrando las cuencas de la Muga y Fluvih y los "Aiguamolls de l'Empordà".

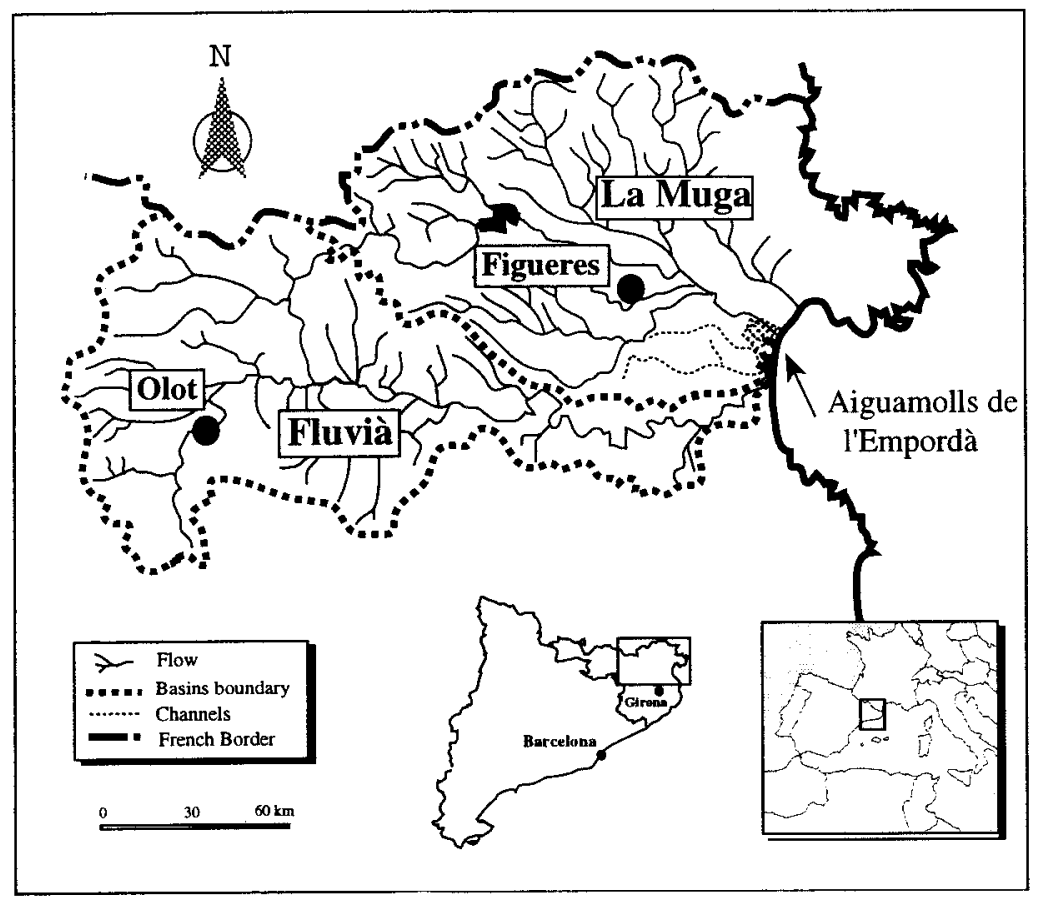


TABLE. 1 Physical characteristics of the study sites in the Empordà wetlands and Muga and Fluvià Basins and the time of sampling. ial s-sand, st-stone. sp-submerged plants and m-mud.

TABLA 1. Morfología de los puntos de estudio en los "Aiguamolls de l'Empordà" y cuencas de la Muga y Fluvià en el momento del muestreo. rai" s-arena. st-piedras. Ipvegetación subacuática y $\mathrm{m}$-fango.

\begin{tabular}{|c|c|c|c|c|c|c|c|c|}
\hline \multicolumn{2}{|c}{ Study site } & Date & $\begin{array}{c}\text { Area } \\
\left(\mathrm{m}^{2}\right)\end{array}$ & $\begin{array}{c}\text { Min. } \\
\text { width } \\
(\mathrm{m})\end{array}$ & $\begin{array}{c}\text { Max. } \\
\text { width } \\
(\mathrm{m})\end{array}$ & $\begin{array}{c}\text { Mean } \\
\text { depth } \\
(\mathrm{m})\end{array}$ & $\begin{array}{c}\text { Length } \\
(\mathrm{m})\end{array}$ & $\begin{array}{c}\text { Bottom } \\
\text { structure }^{\mathrm{a}}\end{array}$ \\
\hline $\begin{array}{c}\text { Fluvià Basin } \\
\text { Castellfollit }\end{array}$ & 30.11 .94 & 640 & 13 & 14 & $1-1,5$ & 50 & $\mathrm{st} \mathrm{-} \mathrm{s}$ \\
Besalú & 29.11 .94 & 4100 & 35 & 38 & $0,4-1,3$ & 110 & $\mathrm{st}$ \\
Vilert & 29.11 .94 & 1340 & 13 & 40 & $0,5-1,5$ & 65 & $\mathrm{st}$ \\
Torroella & 24.11 .94 & 3550 & 15 & 45 & $0,3-1$ & 150 & $\mathrm{~s}-\mathrm{sp}$ \\
Muga Basin & & & & & & & \\
Albanya & 23.11 .94 & 1760 & 14 & 24 & 0,5 & 90 & $\mathrm{st}$ \\
Peralada & 18.11 .94 & 680 & 4,5 & 8 & 0,5 & 120 & $\mathrm{st}-\mathrm{sp}$ \\
Cabanes & 25.11 .94 & 450 & 4 & 13 & 0,5 & 50 & $\mathrm{~s}-\mathrm{sp}$ \\
Vilanova & 18.11 .94 & 1290 & 10 & 22 & $1-1,5$ & 90 & $\mathrm{~s}$ \\
Vilafant & 23.11 .94 & 540 & 7 & 22 & 0,4 & 60 & $\mathrm{sp}$ \\
Castelló & 28.11 .94 & 1020 & 10 & 30 & $1-1,2$ & 80 & $\mathrm{~s}-\mathrm{sp}$ \\
Empordà wetlands & & & & & & & $\mathrm{m}$ \\
Mugueta & 28.11 .94 & 300 & 5 & 5 & 1 & 60 & $\mathrm{~m}$ \\
Rogera & 21.11 .94 & 750 & & - & $0,5-1,5$ & & \\
\hline
\end{tabular}

At all study sites, electrofishing surveys were conducted using the following procedure: sections were blocked off with barrier nets and upon 3 successive electrofishings, performed from the downstream net up to the upstream net. Electric fishing was conducted on foot by one diver in shallow areas using a generator-powered unit (ERREKA model SEINA) that provides fullyrectified triphasic AC (between 50-500 V). The working voltage was generally $200-350 \mathrm{~V}, 2-3 \mathrm{~A}$. The triplicate fishings were carried out trying to keep the effort constant in order to respect the assumptions of catch-effort methods (SEBER, 1982).

All fish was identified, counted, sampled and retained in screened cages until the survey was completed and released thereafter. Sampling included measuring fork lengths $(\mathrm{mm})$ and wet weight (g) (BOHLIN, 1990).

Densities of fish were calculated using catch-depletion data and the removal method (ZIPPIN, 1956 and SEBER, 1982) where maximum-likelihood estimates of $\mathrm{N}$ (population size) and $\mathrm{P}$ (probability of capture) were made for each species separately. Removal is a catch-effort method for closed populations whell a constant sampling effort is applied. The basic assumptions of this method are that: (1) the population is closed during the experiment, (2) the probability of capture in a sample is the same for each individual exposed to capture and, (3) the probability of capture p remains constant from sample to sample. The second and third assumptions are more problematic to be easily assured (MAHON, 1980; SCHNUTE, 1983; BOHLIN et al., 1989) and typically verified by goodness-of-fit statistics (ZIPPIN, 1956). Density estimates were made using the computer program REMOVAL (GARCIA-BERTHOU, 1993), developed to compute all the calculations of the removal method for a population size estimation. The program follows the maximum likelihood methodology, checks the failure conditions, applies the appropiate formula, and displays the estimates of population size and catchability, with their standard deviations and coefficients of variation, and two goodness-of-fit statistics with their significance levels. Where catchability varied significantly between sweeps, density was estimated as the ratio of the total catch to the average catchability of the species (LOBÓN-CERVIÁ, 1990).

Estimated standing crops $(\mathrm{SC})$ were calculated as $(\mathrm{BtCt})+\mathrm{Bc}(\mathrm{N}$ $\mathrm{Ct}$ ), where $\mathrm{Bt}$ is the total weight of fish caught, $\mathrm{Ct}$ is the total number of fish caught and $\mathrm{Bc}(\mathrm{N}-\mathrm{Ct})$ represents the standing crop 
TABLE II. Fish assemblage of La Muga (1) and Fluvià (2) basins and (3) Empordà wetlands.

TABLA II Asociación ictica de las cuencas de La Muga (1) y Fluvià (2) y (3) "Aiguamolls de l'Empordá"

\begin{tabular}{|cllc|}
\hline Code & Fish Species & Common names & Area \\
\hline AAN & Anguilla anguilla (L.) & European eel & $1,2,3$ \\
ELU & Essox lucius (L.) & Pike & 1 \\
BME & Barbus meridionalis (Risso) & Mediterranean barbel & 1,2 \\
CCA & Cyprinus carpio (L.) & Common carp & $1,2,3$ \\
LCE & Leuciscus cephalus (L.) & Chub & 1,2 \\
PPH & Phoxinus phoxinus (L.) & Minnow & 1 \\
RRU & Rutilus rutilus (L.) & Roach & 1 \\
SER & Scardinius erythrophthalmus (L.) & Rudd & 1 \\
GAC & Gasterosteus aculeatus (L.) & Three-spined stickleback & 1 \\
DLA & Dicentrarchus labrax (L.) & Sea bass & 1 \\
LGI & Lepomis gibbosus (L.) & Bluegill sunfish & 1 \\
CHL & Chelon labrosus (Risso) & Thicklipped mullet & 1,2 \\
LRA & Lizaranada (Risso) & Striped mullet & 1,3 \\
MCE & Mugil cephalus (L.) & Grey mullet & 1 \\
BFL & Blennius fluviatilis (Asso) & Freshwater blenny & 1 \\
\hline
\end{tabular}

of the uncaptured fish, estimated by multiplying its number by the mean weight of fish caught in the last electrofishing, as has been applied by differents authors (PENCZAK et al., 1986 and LOBÓN-CERVIA \& UTRILLA, 1992).

A rough estimate of the production has been calculated by multiplying the average biomass by the mean PIB ratio of each fish species (BAGENAL, 1978).

Diversity among species was calculated, using the $\mathrm{H}^{\prime}$ Shannon-Weaver index, derived from their information measure, as $H^{\prime}=-\Sigma$ pi log pi where pi has been estimated by the ratio ni $/ \mathrm{N}$

\section{RESULTS}

\section{Fish assemblage}

Species recorded at 12 study sites on the Muga and Fluvih rivers, by electrofishing catches. are shown in Table II. Size selectivity of electrofishing gear may induce a lack of species with small total size, like Blennius fluviatilis (Asso), and Gasterosteus aculeatus (L.) at some stations. A code for each species based on its scientific name is used in order to simplify the presentation of different results.

The Muga Basin presents a fish assemblage of 15 detected species, in contrast with the 3 fish species of the Empordh wetlands and the 5 species of the Fluvih Basin. In order to compare the different study sites in terms of fish species richness a Cluster Analysis has been peformed, obtaining the classification presented in dendrogram of Figure 2.

\section{Populations size, biomass and production}

Stock density, standing crop and production estimates at the sites after three catches are presented in Table 111 for the Muga Basin, in Table IV for the Empordà Wetlands and Table V for the Fluvià Basin. Out of 46 density assessments, the method of Moran and Zippin (SEBER, 1982) modified in 3 samples by JUNGE \& LIBOSVARSKÎ (1965), could be applied in 17 cases. The factor limiting the application of the removal method (in 29 cases) was the low number of fish of a given species at a particular site, and the failure of the method because the biggest amount of catches ocurred in $\mathrm{C} 2$ or $\mathrm{C} 3$ (second and third sweeps). 
TABLE III Populations sıze. biomass and production obtained for sites and their fish species for La Muga Basin. N are the estimated number of fish by station: $\mathrm{p}$. catchability: $s(\mathrm{~N})$ : Standard deviation of $\mathrm{N}: \mathrm{s}(\mathrm{p})$ ): Standard error of p. Total biomass. Standing Crop. Biomass and Production as fresh weight (g).

TABLA III. Tamaño de la población, biomasa y producción estimadas para cada punto de muestreo y especie en la cuenca de la Muga. N es el número estimado de peces para cada punto; p: capturabilidad; $\mathrm{s}(\mathrm{N})$ : desviación estandard de $\mathrm{N} ; \mathrm{s}(\mathrm{p})$ : desviación estandard de $\mathrm{p}$. Biomasa total. standing crop, biomasa y producción expresadas en gramos de peso fresco.

\begin{tabular}{|c|c|c|c|c|c|c|c|c|c|c|}
\hline Study site & Species & $\begin{array}{l}\text { Total } \\
\text { catch }\end{array}$ & $\mathrm{N}$ & $\mathrm{p}$ & $s(N)$ & $S(p)$ & $\begin{array}{c}\text { Total } \\
\text { biomass } \\
\text { (g) }\end{array}$ & $\begin{array}{c}\text { Standing } \\
\text { Crop } \\
\text { (g) }\end{array}$ & $\begin{array}{l}\text { Biomass } \\
\left(\mathrm{g} \cdot \mathrm{m}^{-2}\right)\end{array}$ & $\begin{array}{l}\text { Production } \\
\left(\mathrm{g} \cdot \mathrm{m}^{-2} \mathrm{y}^{-1}\right)\end{array}$ \\
\hline Albanyà & $\begin{array}{l}\text { BME } \\
\text { LCE }\end{array}$ & $\begin{array}{c}60 \\
3 \\
63\end{array}$ & $\begin{array}{c}60,1 \\
3,1 \\
63,1\end{array}$ & $\begin{array}{l}0,89 \\
0,71\end{array}$ & $\begin{array}{l}0,28 \\
0,36\end{array}$ & $\begin{array}{l}0,04 \\
0,28\end{array}$ & $\begin{array}{c}1045 \\
40 \\
\mathbf{1 0 8 5}\end{array}$ & $\begin{array}{c}1045 \\
40 \\
\mathbf{1 0 8 5}\end{array}$ & $\begin{array}{c}0,594 \\
0,023 \\
\mathbf{0 , 6 1 6}\end{array}$ & $\begin{array}{l}1,11 \\
0,03 \\
\mathbf{1}, \mathbf{1}\end{array}$ \\
\hline Cabanes & $\begin{array}{l}\text { AAN } \\
\text { BME } \\
\text { ELU } \\
\text { LCE } \\
\text { RRU }\end{array}$ & $\begin{array}{c}105 \\
2 \\
2 \\
5 \\
208 \\
\mathbf{3 2 2}\end{array}$ & $\begin{array}{c}120,9 \\
2 \\
2 \\
7 \\
214,3 \\
\mathbf{3 4 6 , 3}\end{array}$ & $\begin{array}{c}0,49 \\
1 \\
\text { I } \\
0,72 \\
0,69\end{array}$ & 8,50 & 0,07 & $\begin{array}{c}1780 \\
90 \\
645 \\
70 \\
8010 \\
\mathbf{1 0 5 9 5}\end{array}$ & $\begin{array}{c}8005,5 \\
90 \\
645 \\
98 \\
8253 \\
\mathbf{1 7 0 9 1 , 5}\end{array}$ & $\begin{array}{c}17,8 \\
0,2 \\
1,4 \\
0,2 \\
18,3 \\
37,9\end{array}$ & $\begin{array}{c}20,5 \\
0,4 \\
1 \\
0,2 \\
20,5 \\
42,6\end{array}$ \\
\hline Peralada & $\begin{array}{l}\text { AAN } \\
\text { BME } \\
\text { CCA } \\
\text { LCE } \\
\text { RRU }\end{array}$ & $\begin{array}{c}21 \\
25 \\
2 \\
25 \\
8 \\
\mathbf{8 1}\end{array}$ & $\begin{array}{c}22,7 \\
48,4 \\
2,1 \\
25,7 \\
12,8 \\
\mathbf{1 1 1 , 6}\end{array}$ & $\begin{array}{c}0,58 \\
0,21 \\
0,64 \\
0,7 \\
0,62\end{array}$ & $\begin{array}{c}34,42 \\
- \\
-\end{array}$ & 0,20 & $\begin{array}{c}2020 \\
425 \\
1315 \\
960 \\
400 \\
\mathbf{5 1 2 0}\end{array}$ & $\begin{array}{c}2077,5 \\
822,5 \\
1315 \\
986,5 \\
640 \\
\mathbf{5 8 4 1 , 5}\end{array}$ & $\begin{array}{l}3,1 \\
1,2 \\
1,9 \\
1,5 \\
0,9 \\
8,6\end{array}$ & $\begin{array}{c}3,5 \\
2,3 \\
1 \\
1,7 \\
1,1 \\
9,5\end{array}$ \\
\hline Vilanova & $\begin{array}{l}\text { AAN } \\
\text { CCA } \\
\text { LGI }\end{array}$ & $\begin{array}{c}67 \\
45 \\
4 \\
\mathbf{1 1 6}\end{array}$ & $\begin{array}{c}72,4 \\
47,2 \\
4,6 \\
\mathbf{1 2 4 , 1}\end{array}$ & $\begin{array}{c}0,58 \\
0,64 \\
0,5\end{array}$ & - & & $\begin{array}{c}9495 \\
66780 \\
40 \\
\mathbf{7 6 3 1 5}\end{array}$ & $\begin{array}{c}10254,6 \\
70044,8 \\
40 \\
\mathbf{8 0 3 3 9 , 4}\end{array}$ & $\begin{array}{c}7,9 \\
54,3 \\
0,03 \\
\mathbf{6 2 , 3}\end{array}$ & $\begin{array}{c}9,1 \\
27,1 \\
0,03 \\
\mathbf{3 6 , 3}\end{array}$ \\
\hline Vilafant & $\begin{array}{l}\text { AAN } \\
\text { BME } \\
\text { CCA } \\
\text { LCI } \\
\text { LCE } \\
\text { RRU }\end{array}$ & $\begin{array}{c}14 \\
1 \\
76 \\
4 \\
2 \\
1 \\
\mathbf{9 8}\end{array}$ & $\begin{array}{c}50,8 \\
2 \\
122,9 \\
5,3 \\
2,7 \\
1,6 \\
\mathbf{1 8 5 , 3}\end{array}$ & $\begin{array}{c}0,10 \\
0,7 \\
0,27 \\
0,5 \\
0,7 \\
0,62\end{array}$ & $\begin{array}{c}132,29 \\
-\end{array}$ & 0,29 & $\begin{array}{c}1690 \\
35 \\
52200 \\
35 \\
10 \\
70 \\
\mathbf{5 4 0 4 0}\end{array}$ & $\begin{array}{c}6137,1 \\
70 \\
84399,1 \\
46,6 \\
13,5 \\
112 \\
\mathbf{9 0 7 7 8 , 4}\end{array}$ & $\begin{array}{c}11,4 \\
0,1 \\
156,3 \\
0,1 \\
0,02 \\
0,20 \\
\mathbf{1 6 8 , 1}\end{array}$ & $\begin{array}{c}13,1 \\
0,2 \\
78,1 \\
0,1 \\
0,03 \\
0,2 \\
\mathbf{9 1 , 8}\end{array}$ \\
\hline Castelló & $\begin{array}{l}\text { AAN } \\
\text { CHL } \\
\text { CCA } \\
\text { DLA } \\
\text { LGI } \\
\text { LCE } \\
\text { LRA } \\
\text { MCE } \\
\text { RRU }\end{array}$ & $\begin{array}{c}46 \\
17 \\
49 \\
27 \\
1 \\
28 \\
199 \\
18 \\
2 \\
\mathbf{3 8 7}\end{array}$ & $\begin{array}{c}47,6 \\
17 \\
49,4 \\
28,6 \\
2 \\
29,5 \\
203,1 \\
18,7 \\
2,2 \\
\mathbf{3 9 8}\end{array}$ & $\begin{array}{c}0,68 \\
0,94 \\
0,80 \\
0,61 \\
0,5 \\
0,63 \\
0,72 \\
0,66 \\
0,56\end{array}$ & $\begin{array}{c}1,87 \\
- \\
- \\
0,74\end{array}$ & $\begin{array}{l}0,05 \\
0,06 \\
0,11 \\
0,11\end{array}$ & $\begin{array}{c}6565 \\
12295 \\
82090 \\
24560 \\
5 \\
365 \\
140425 \\
11825 \\
8 \\
\mathbf{2 7 8 1 3 8}\end{array}$ & $\begin{array}{c}6787,6 \\
12295 \\
82090 \\
26046,2 \\
10 \\
384,5 \\
143290 \\
12291,4 \\
43,4 \\
\mathbf{2 8 3 2 3 8 , 2}\end{array}$ & $\begin{array}{c}6,6 \\
12,1 \\
80,5 \\
25,5 \\
0,01 \\
0,4 \\
140,5 \\
12,05 \\
0,04 \\
\mathbf{2 7 7 , 7}\end{array}$ & $\begin{array}{c}7,6 \\
6,03 \\
40,2 \\
10,2 \\
0,009 \\
0,4 \\
56,2 \\
4,8 \\
0,048 \\
\mathbf{1 2 5 , 6}\end{array}$ \\
\hline
\end{tabular}




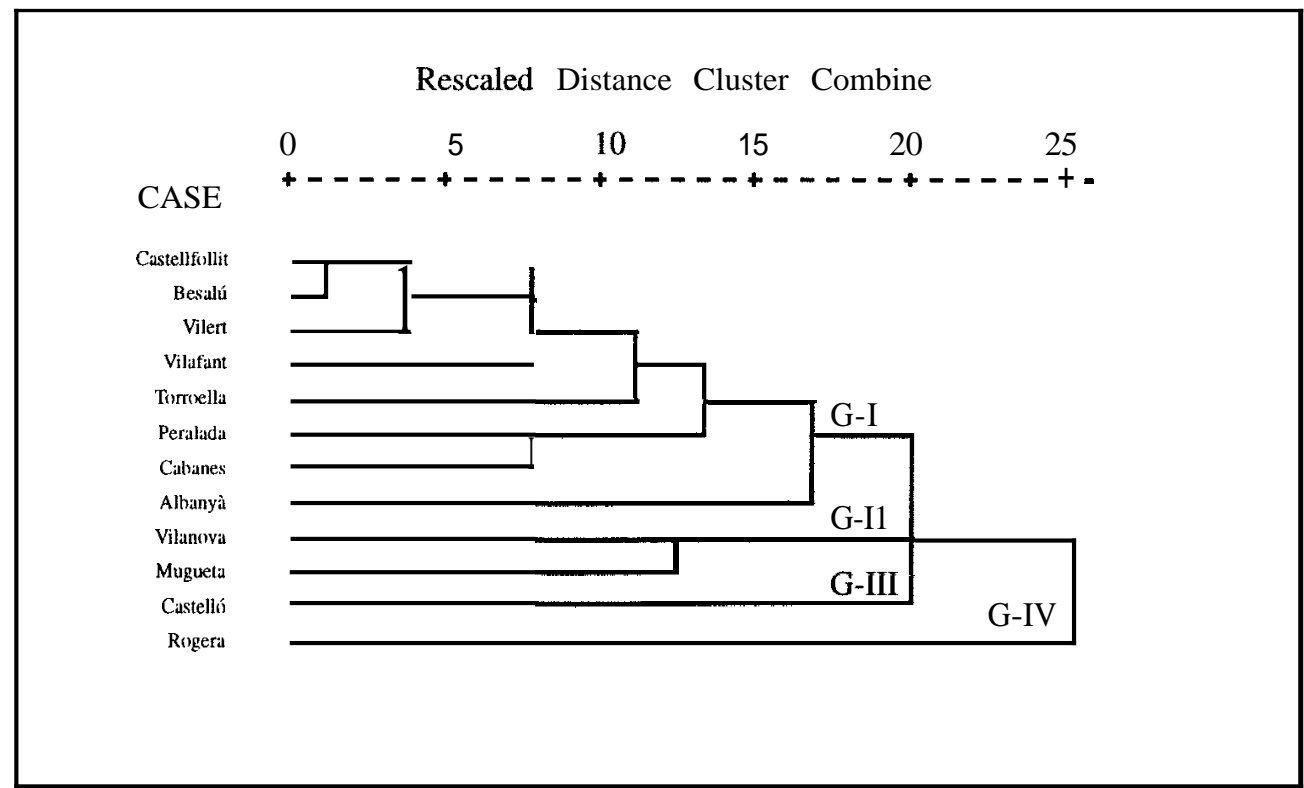

FIGURE 2. Dengrogram using Average Linkage between groups (study sites) showing the relations between site? and groups of sites using the similarity ratio.

FIGURA 2. Dendrograma de clasificación calculado a partir de In relación media entrc grupos (puntos de muestreo) mostrando la relación entre puntos y grupos de puntos usando el índice de similitud.

Production was estimated indirectly by multiplying the average biomass by the mean $\mathrm{P} / \mathrm{B}$ ratio. This information has been compiled trom published sources (MANN \& PENCZAK, 1986 and JORGENSEN, 1978), which provide a revision of turnover ratio for non-salmonid fishes in European rivers. We applied the following values: AAN 1,15; ELU 0,7; BME 1,87; CCA 0,5; RRU 1,12; SER 1,12; DLA 0,4; LGI 0,95; CHL 0,5; LRA 0,4 and MCE 0,4 .

Table V1 presents a summary of REMOVAL program output results with a classification of the size population estimate for each site and those fish species in which the removal method could be applied, those in which the estimate was statistically significant and those in which there was a failure.

The density assessment at sites which belong to both significant and failure status, has been obtained by multiplying total catches by catchability (BOHLIN \& COWX, 1990). Confidence intervals for the population density are not provided due to high values of standard error.

Due to heterogeneity among study sites, mean standing crops and productions have not been calculated. La Muga river presents a range of standing crop variability of 1,085-283,238 kg. 0,616-277,6 g m ${ }^{2}$ biomass, and 1 ,136-125,637 g.m ${ }^{2} . y^{\prime}$ production . The Fluvià River presents standing crop ranges between 31,191 and $471.561,8,786-351,911 \mathrm{~g} \cdot \mathrm{m}^{-2}$ biomassa, and 4,49436,05 g.m ${ }^{2} . y^{\prime}$ production. These ranges correspond to a gradient between upstream waters and currents flowing into the wetlands.

\section{Density}

Species richness as total number of species, fish density expressed as ind.ha' and diversity values for study sites are given in Figure 3. Diversity was calculated according to the Shannon-Weaver meaxure of information. The Muga river presents a higher richness due to the presence of the Boadella reservoir, with a lot of introduced species and the influx of sea fish. Values increase along the river as it becomes wider and it finds is a higher diversity of habitats. The Fluvià river presents a more homogeneus pattern with an important change in Vilert, where fish density is very high and this involves biomass and production maxima.

\section{Length-frequency comparisons}

Size structure was examined due to its direct usefulness in assessing the community and population structure, and because of the fact that any size selectivity imposed by electrofishing can influence other estimates such as density and biomass (MAHON, 1980).

We have observed a size selection with a lack of young fish. Despite this evidence, we can not use this representation to determine the minimun size caught or size selection of larger fish since there is also an effect on population structure.

A summary of the major variables measured and its values is shown in Table VII for each fish species detected. 
TABLE IV. Population size. biomass and production obtained from each site and its fish species in the Empordh wetlands, $\mathrm{N}$ is the estimated number of fish per station, $\mathrm{p}$ : catchability; $s(\mathrm{~N})$ : Standard deviation of N; S(p): Standard error of p. Total biomass, Standing Crop, Biomass and Production as fresh weight $(\mathrm{g})$.

TABLA IV. Tamaño de la población, biornasa y producción estimadas para cada punto de muestreo y especie en los Aiguamolls de l'Empordà. N es el número estimado de peces para cada punto; p: capturabilidad: $s(\mathrm{~N})$; desviación estandnrd de $\mathrm{N} ; \mathrm{s}(\mathrm{p})$ : desviacidn estandard de p. Biomasa total, standing crop, biomasa y producción expresadas en gramos de peso fresco

\begin{tabular}{|c|c|c|c|c|c|c|c|c|c|c|}
\hline Study site & Species & $\begin{array}{l}\text { Total } \\
\text { catch }\end{array}$ & $\mathrm{N}$ & $\mathrm{p}$ & $s(N)$ & $S(p)$ & $\begin{array}{c}\text { Total } \\
\text { biomass } \\
(\mathrm{g})\end{array}$ & $\begin{array}{l}\text { Standing } \\
\text { Crop } \\
(\mathrm{g})\end{array}$ & $\begin{array}{l}\text { Biomass } \\
\left(\mathrm{g} \cdot \mathrm{m}^{-2}\right)\end{array}$ & $\begin{array}{l}\text { Production } \\
\left(\text { g. } \mathrm{m}^{-2} \mathrm{y}^{-1}\right)\end{array}$ \\
\hline Mugueta & $\begin{array}{l}\text { AAN } \\
\text { CCA }\end{array}$ & $\begin{array}{l}4 \\
2 \\
6\end{array}$ & $\begin{array}{l}4 \\
2 \\
6\end{array}$ & $\begin{array}{c}\text { I } \\
0,64\end{array}$ & & & $\begin{array}{c}410 \\
1315 \\
\mathbf{1 7 2 5}\end{array}$ & $\begin{array}{c}410 \\
1315 \\
\mathbf{1 7 2 5}\end{array}$ & $\begin{array}{l}1,4 \\
4,4 \\
\mathbf{5 , 7}\end{array}$ & $\begin{array}{l}1,6 \\
2,2 \\
\mathbf{3}, \mathbf{8}\end{array}$ \\
\hline Rogera & $\begin{array}{l}\text { CCA } \\
\text { LRA }\end{array}$ & $\begin{array}{l}39 \\
41 \\
80\end{array}$ & $\begin{array}{c}40,9 \\
47,4 \\
\mathbf{8 8 , 3}\end{array}$ & $\begin{array}{l}0,64 \\
0,49\end{array}$ & & & $\begin{array}{c}62735 \\
13535 \\
\mathbf{7 6 2 7 0}\end{array}$ & $\begin{array}{c}65791,3 \\
15647,7 \\
81439,02\end{array}$ & $\begin{array}{c}87,7 \\
20,9 \\
\mathbf{1 0 8 , 6}\end{array}$ & $\begin{array}{c}43,9 \\
8,3 \\
52,2\end{array}$ \\
\hline
\end{tabular}

TABLE V. Population size, biomass and production obtained from each site and its fish species in the Fluvià Basin, $\mathrm{N}$ is the estimated number of fish per station; p: catchability, $\mathrm{s}(\mathrm{N})$ : Standard deviation of $\mathrm{N} ; \mathrm{s}(\mathrm{p})$ : Standard error of $\mathrm{p}$. Total biomass, Standing Crop, Biomass and Production as fresh weight (g).

TABLA V. Tamaño de la población, biomasa y produccion estimadas para cada punto de muestreo y especie en la cuenca del Fluvià. $\mathrm{N}$ es el numero estimado de peces para cada punto; p: capturabilidad; $s(\mathrm{~N})$ : desviacion estandard de $\mathrm{N} ; \mathrm{s}(\mathbf{p})$ : desviación estandard de p. Biomasa total. standing crop. biomasá y produccion expresadas en gramos de peso fresco.

\begin{tabular}{|c|c|c|c|c|c|c|c|c|c|c|}
\hline Study site & Species & $\begin{array}{l}\text { Total } \\
\text { catch }\end{array}$ & $\mathrm{N}$ & $\mathrm{p}$ & $\mathrm{s}(\mathrm{N})$ & $S(p)$ & $\begin{array}{c}\text { Total } \\
\text { biomass } \\
(\mathrm{g})\end{array}$ & $\begin{array}{c}\text { Standing } \\
\text { Crop } \\
(\mathrm{g}) \\
\end{array}$ & $\begin{array}{c}\text { Biomass } \\
\left(\mathrm{g} \cdot \mathrm{m}^{-2}\right)\end{array}$ & 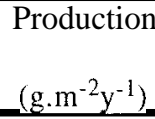 \\
\hline Castellf. & $\begin{array}{l}\text { AAN } \\
\text { BME } \\
\text { CCA } \\
\text { LCE }\end{array}$ & $\begin{array}{c}2 \\
65 \\
108 \\
41 \\
\mathbf{2 1 6}\end{array}$ & $\begin{array}{c}4 \\
81,6 \\
169 \\
64,6 \\
\mathbf{3 1 9 , 2}\end{array}$ & $\begin{array}{l}0,5 \\
0,4 \\
0,3 \\
0,3\end{array}$ & $\begin{array}{r}11,1 \\
1,9\end{array}$ & $\begin{array}{c}0,09 \\
0,1\end{array}$ & $\begin{array}{c}690 \\
2575 \\
108160 \\
8215 \\
\mathbf{1 1 9 6 4 0}\end{array}$ & $\begin{array}{c}1380 \\
3064,6 \\
169240,3 \\
10946,1 \\
\mathbf{1 8 4 6 3 1}\end{array}$ & $\begin{array}{c}2,1 \\
4,8 \\
264,4 \\
17,1 \\
\mathbf{2 8 8 , 5}\end{array}$ & $\begin{array}{c}2,5 \\
8,9 \\
132,2 \\
19,7 \\
\mathbf{1 6 3 , 3}\end{array}$ \\
\hline Besalú & $\begin{array}{l}\text { AAN } \\
\text { BME } \\
\text { CCA } \\
\text { LCE }\end{array}$ & $\begin{array}{c}38 \\
67 \\
106 \\
53 \\
\mathbf{2 6 4}\end{array}$ & $\begin{array}{c}42 \\
72,1 \\
110,4 \\
54 \\
\mathbf{2 7 8 , 5}\end{array}$ & $\begin{array}{l}0,5 \\
0,6 \\
0,6 \\
0,7\end{array}$ & $\begin{array}{l}3,7 \\
3,8\end{array}$ & $\begin{array}{c}0,1 \\
0,07\end{array}$ & $\begin{array}{c}9760 \\
1745 \\
75645 \\
6925 \\
\mathbf{9 4 0 7 5}\end{array}$ & $\begin{array}{c}10787,4 \\
1878,3 \\
78785 \\
7053 \\
\mathbf{9 8 5 0 3 , 7}\end{array}$ & $\begin{array}{c}2,6 \\
0,5 \\
19,2 \\
1,7 \\
\mathbf{2 4 , 0 2}\end{array}$ & $\begin{array}{c}3,02 \\
0,8 \\
9,6 \\
2,0 \\
\mathbf{1 5 , 5}\end{array}$ \\
\hline Vilert & $\begin{array}{l}\text { AAN } \\
\text { BME } \\
\text { CCA } \\
\text { LCE } \\
\text { SER }\end{array}$ & $\begin{array}{c}116 \\
1045 \\
25 \\
3965 \\
568 \\
\mathbf{5 7 1 9}\end{array}$ & $\begin{array}{c}116 \\
1045 \\
25 \\
3965 \\
568 \\
\mathbf{5 7 1 9}\end{array}$ & $\begin{array}{l}\text { I } \\
1 \\
1 \\
1 \\
1\end{array}$ & & & $\begin{array}{c}13920 \\
81635 \\
25025 \\
334687 \\
16294 \\
\mathbf{4 7 1 5 6 1}\end{array}$ & $\begin{array}{c}13920 \\
81635 \\
25025 \\
334687 \\
16294 \\
\mathbf{4 7 1 5 6 1}\end{array}$ & $\begin{array}{c}10,4 \\
60,9 \\
18,7 \\
249,8 \\
12,2 \\
\mathbf{3 5 1 , 9}\end{array}$ & $\begin{array}{c}11,9 \\
113,9 \\
9,4 \\
287,2 \\
13,6 \\
\mathbf{4 3 6}\end{array}$ \\
\hline Torroella & $\begin{array}{l}\text { AAN } \\
\text { CHL } \\
\text { CCA } \\
\text { LCE }\end{array}$ & $\begin{array}{c}23 \\
11 \\
16 \\
3 \\
53\end{array}$ & $\begin{array}{c}23,03 \\
11,715 \\
16,02 \\
4,1 \\
\mathbf{5 4 , 9}\end{array}$ & $\begin{array}{l}0,9 \\
0,6 \\
0,9 \\
0,7\end{array}$ & $\begin{array}{l}0,2 \\
1,3 \\
0,1\end{array}$ & $\begin{array}{c}0,06 \\
0,1 \\
0,08\end{array}$ & $\begin{array}{c}570 \\
9270 \\
21345 \\
5 \\
\mathbf{3 1 1 9 0}\end{array}$ & $\begin{array}{c}570 \\
9270 \\
21345 \\
6,8 \\
\mathbf{3 1 1 9 2}\end{array}$ & $\begin{array}{c}0,16 \\
2,6 \\
6,01 \\
0,002 \\
\mathbf{8 , 8}\end{array}$ & $\begin{array}{c}0,18 \\
1,3 \\
3,006 \\
0,002 \\
\mathbf{5}\end{array}$ \\
\hline
\end{tabular}


TABLE VI. Summary of REMOVAL program output results: classification of sites and their fish species acording to goodness-of-fit statistics of population size estimate. Estimation of population size not significant.- 1: Method of Moran (1951) and Zippin (1956) modified for 2 samples by Seber-Le Cren (1967); 2: Method of Moran (1951) and Zippin (1956) modified for 7 sample5 by Jungè \& Libosvêrsky (1965); Estimation of population sıze significant: 5\%: Significant at the 5\% level; I\%: Significant at the

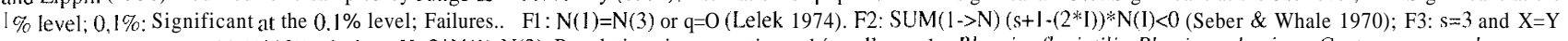
or $\left(\left(\mathrm{Y}^{*} * 2\right)+(6 * \mathrm{X} * \mathrm{Y})-\left(3 *\left(\mathrm{X}^{*} * 2\right)\right)<0\right.$ where $\mathrm{X}=2 * \mathrm{~N}(1)-\mathrm{N}(3)$. Population size not estimated (small sample: Blennius fluviatilis, Phoxinus phoxinus, Gasterosteus aculeatus.

TABLA VI Resumen de los resultados obtenidos aplicando el programa REMOVAL: Clasificación de los puntos de estudio y de las especies presentes en función de la bondad de ajustamiento de la estima de la población. Estimación no significativa- 1: Métode de Moran (1951) y Zippin (1956) modificado para dos muestras por Seber-Le Cren (1967); 2: Métode de Moran (1951) y Zippin (1956) modificado para 3 capturas por Jungt \& Libosvêrsky (1965); Estimación significativa.- Significativa al nivel 5\%; 1\%: Significativa al nivel I\%; 0.1\%: Significativa al nivel 0.1\%. Fallos. FI: N(1)=N(3) o q=O (Lelek 1974); F2: SUM(I->N (s+1-(2*I))*N(I)<0 (Seber \&Whale 1970): F3: $s=3$ y $\mathrm{X}=\mathrm{Y}$ o $\left(\left(\mathrm{Y}^{*} * 2\right)+\left(6^{*} \mathrm{X}^{*} \mathrm{Y}\right)-\left(\mathrm{e}^{*}\left(\mathrm{X}^{* *} 2\right)\right)<0\right.$ donde $\mathrm{X}=2 * \mathrm{~N}(1)+\mathrm{N}(2)$ y $\mathrm{Y}=2 * \mathrm{~N}(1)-\mathrm{N}(3)$. Tamaño de la población no estimada (muestras pequeñas): Blennius fltwiafilis, Phoximus phoximus, Gasterosteus aculetatus.

\begin{tabular}{|c|c|c|c|c|c|c|c|c|}
\hline \multirow{2}{*}{$\begin{array}{c}\text { Sample } \\
\text { sites }\end{array}$} & \multicolumn{2}{|c|}{ not significant. } & \multicolumn{3}{|c|}{ significant at the level } & \multicolumn{3}{|c|}{ Failures } \\
\hline & Method 1 & Method 2 & $5 \%$ & $1 \%$ & $0,1 \%$ & $\mathrm{~F} 1$ & F2 & F3 \\
\hline Albanya & & BME LCE & & & & & & \\
\hline Peralada & & BME & & & & & AANCCA & LCE \\
\hline Cabanes & & $\mathrm{AAN}$ & RRU & & LCE & BME ELU & & \\
\hline Vilanova & & & & & & & AAN CCA & LGI \\
\hline Vilafant & & AAN & CCA & & & LCE & LGI & BME \\
\hline Castelló & & $\begin{array}{c}\text { CHLCCA } \\
\text { DLALCE } \\
\text { RRU }\end{array}$ & AANMCE & LRA & & & & \\
\hline Mugueta & & & & & & AANCCA & & \\
\hline Rogera & & & LRA & & & AAN CCA & & CCA \\
\hline Castellfollit & & BMELCE & CCA & & & & & AAN \\
\hline Besalu & & AAN BME & & & CCA LCE & & & \\
\hline Vilert & $\begin{array}{c}\text { AAN BME } \\
\text { CCA LCE } \\
\text { SER }\end{array}$ & & & & & & & \\
\hline Torroella & & $\begin{array}{c}\text { AANCHL } \\
\text { CCA }\end{array}$ & & & & LCE & & \\
\hline
\end{tabular}


TABLA VII. Descriptive statistics for fish species detected: mean (minimum - maximun) of weight (g) and lenght (cm), with standard deviation ( $\mathrm{s}$ ) of mean. Total inumb. Number of valid observations (listwise)

TABLA VII. Estadísticos descripttvos para las especies íctiques detectadas: media (mínimo-máximo) del peso ( $\mathrm{g}$ ) y longitud furcal (cm), con la desviación estándard (s) dr la media. Total Numb.: Numero de observaciones válidas.

\begin{tabular}{|lccccc|}
\hline \multicolumn{1}{c}{ Fish } & Total & WEIGHT & \multicolumn{2}{c|}{ LENGTH } \\
specie & Numb. & Mean & S & Mean & s \\
\hline Anguilla anguilla (L.) & 320 & $36,8(15-71)$ & 11,19 & $119,9(5-750)$ & 133,2 \\
Essox lucius (L.) & 2 & $35(33-37)$ & 2,83 & $322,5(280-365)$ & 60,10 \\
Barbus meridionalis (Risso) & 220 & $11,55(6-22)$ & 3,03 & $26,75(5-160)$ & 25,48 \\
Cyprinus carpio (L.) & 443 & $35,68(6-57)$ & 10,21 & $1071(5-3575)$ & 696,6 \\
Leuciscus cephalus (L.) & 160 & $17,59(6-35)$ & 7,56 & $108,1(0-520)$ & 131,9 \\
Phoxinus phoxinus (L.) & 4 & $4(1-9)$ & 4,36 & & \\
Rutilus rutilus (L.) & 219 & $13,56(7-22)$ & 3,15 & $39,09(5-180)$ & 30,17 \\
Scardinius erythrophthalmus(L.) & 1 & 31 & & & \\
Gasterosteus aculeatus (L.) & 1 & 4 & & & \\
Dicentrarchus labrax (L.) & 27 & $38,37(26-60)$ & 9,5 & $909,6(200-3100)$ & 809,5 \\
Lepomis gibbosus (L.) & 9 & $7,67(4-9)$ & 1,73 & $8,89(0-20)$ & 6,01 \\
Chelon labrosus (Risso) & 28 & $36,79(27-47)$ & 4,04 & $770,2(400-1630)$ & 262,8 \\
Liza ramada (Risso) & 240 & $35,75(9-49)$ & 8,49 & $641,5(10-1390)$ & 275,6 \\
Mugil cephalus (L.) & 18 & $36,28(23-45)$ & 4,32 & $656,9(145-1310)$ & 235,3 \\
Blennius fluviatilis (Asso) & 1 & & & & \\
\hline
\end{tabular}

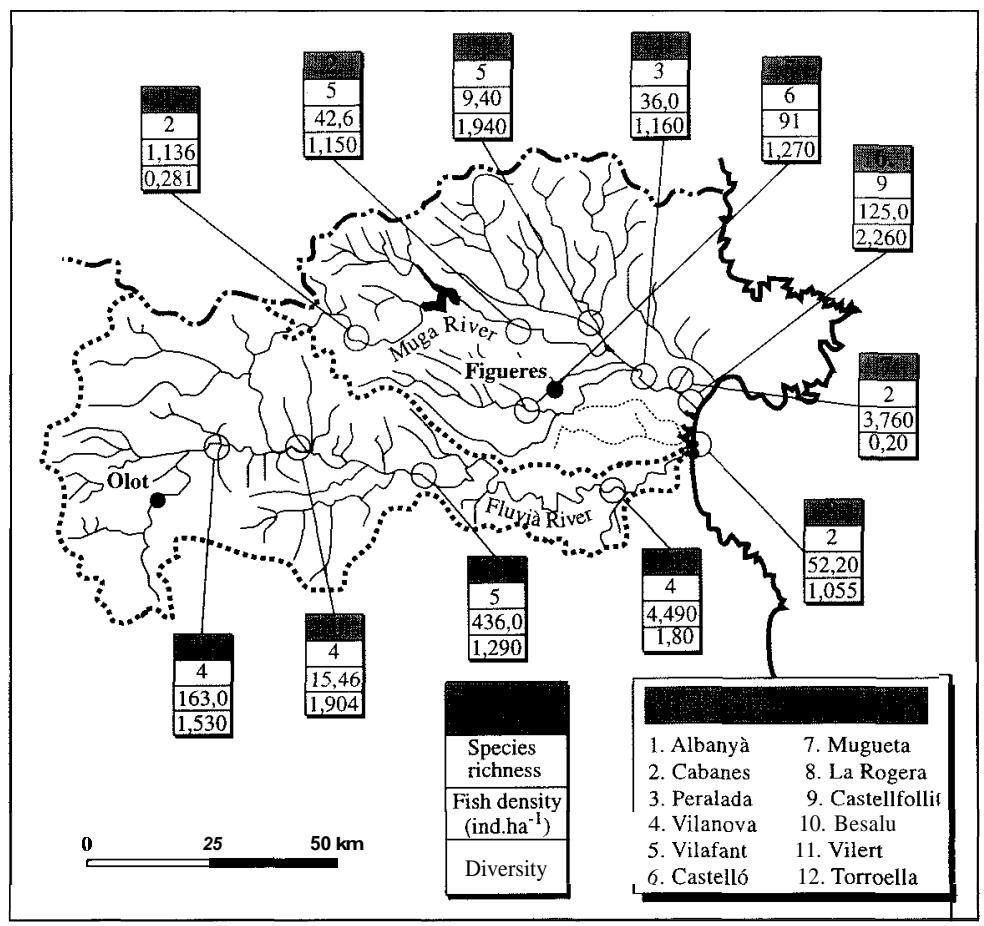

FIGURE 3. Species richness as total number of species, fish density expressed as ind ha 'and diversity values for study sites. See text for more information.

FIGURA 3. Riqueza especifica (como número total de especies), densidad piscicola y diversidad para cada punto de muestreo. Ver texto para más información. 


\section{DISCUSSION}

Two are the limitations which can be born in mind when the results are examinated. The first, that the study area is so extensive that it would imply a larger sampling effort. This has been restricted to 12 sites assigned to the Muga and Fluvià Basins and the Empordà wetlands with the assumption that each site represents the stretch. The traits of the study sites are the same up to the middle of the distance from the follow-up point, both downstream and upstream. On the other hand, electrofishing surveys were conducted all at a time, in November, so that production will be estimated indirectelly from the $\mathrm{P} / \mathrm{B}$ ratio of each fish species. This ratio takes different values in accordance with the environment where the fish species is found.

Early surveys carried out in the study area (SOSTOA, 1990) show a fish species richness that coincides with our own results, with a maximum diversity (Shannon-Weaver index) never higher than 2 bits. In a basin, in general, complexity increases (as for species richness and diversity) from head to mouth, in correlation with altitude, slope and river order (SOSTOA, 1990). The fish species composition in both rivers differs slightly, being the European eel, the mediterranean barbel and the chub the clear dominant ones at headwaters, and common carps, rudds, the bluegill sunfish and the European eel downstream; at some locations near the mouth, marine fish species have been detected (sea bass, thicklipped mullet, striped mulled and grey mullet). The following are accompanying species, characterized by a low occurrence: pike, minnow, roach and freshwater blenny.

Dendrogram of Figure 2 shows the relations between sites where hierarchical structure is indicated by the branching pattern using Average Linkage between groups (study sites). The dice (or Czekanowski or Sorenson) similarity measure has been used because it is a matching coefficient measure in which joint absences are excluded in both the numerator and the denominator and double weight is given to matches. Joint absences are not suitable when removals are not exhaustive, because the absence of species can be due to a low efficiency of caughts. Moreover, double weight of joint presences increases similarity values when complete matching can not be expected due to a low number of species and an inefficient sampling.

This analysis classifies sites h 14 groups distinguished, respectively, from top to bottom, by basins and sites located at the same distance from the sea (GI), sites next to the mouth with a low numbelof species (GII), presence of a large number of species (GIII, Case 6-Castello) and la Rogera (GIII) as the most different with only two species and no joint presence.
After having applied the Removal method over 46 density assessments, 17 estımates come out non-significant. In 29 cases we obtained bad estimations as a consequence of small samplings and method failures. In these cases the model for tested populations is not valid by a violation of basic assumptions.

Three possible sources of low efficiency or error can be identified: (1) differences between species, which are called biotic factors, (2) locality characteristics such as visibility, overhanging vegetation, substrates, called abiotic factors, and (3) the experience of electrofishers. It is evident that there is a large proportion of unexplained variability in error as well. The accuracy of the removal method applied in electric fishing has been assessed by different authors (BOHLIN, 1990) in order to improve the efficiency of catches.

Failures occur in all species and places (except Albanya) when fishes were caught in a single removal. Low density probably produced this result in those places in which the habitat was the least suitable to determinate fish species (Mediterranean barbels in Vilafant or European eels in Castellfollit, for instance).

Differences among species with regard to behavioral, physiological and morphological features (biotic factors) could affect capture. Significant estimations or failures were obtained for fish species such as common carp (Vilafant, Besalli), striped mullet (Castelló) or chub (Besalli), which form shoals.

Abiotic factors which can affect the electrofishing efficiency are river width and presence of submerged plants. Efficiency of electrofishing was found to decrease with an increasing river width (KENNEDY et al., 1981); this occurs in Torroella and Castelló Besalli, where the riverbed bottom presented an large area occupated by vegetation.

Large fishes are always assumed to be more susceptible to the electric current and therefore more catchable than the small ones. It is generally expected that selectivity will manifest itself as a decrease in mean size in successive catches. Whether large or small fishes are more vulnerable and removed first, decreasing catchability and consequent underestimation will result (ZALEWSKI, 1983). To determine the extent to which size-selectivity ocurred, correlations between mean weight and catch number were calculated. Only five cases show a correlation: the European eel in Cabanes and Perelada, the Mediterranean barbel in Besalli and Castellfollit and the chub in Castellfollit.

In any case, the most important factor that has determined electrofishing efficiency has been the variability of the fishing effort. After catchability among removal were examined. we have noticed that it takes different values when we would have 
expected it to be constant if the fishing etfort had been unchanged. Consequences of this variability are a low absolute catchability, getting small samples and an irregular decline in the population removed. This is the case of the bad estimations from Castello (European eel, grey mullet), Vilafant (common carp), Castellfollit (common carp), Besalú (common carp, chub) or Cabanes (roach).

The density of fish at the surveyed locations varies from 1,13 to 163 ind ha'. If these results are referred to the components of the ichthyofauna, it can be stated that the highest density of fish and biomass states were recolded from locations in which the common carp and the chub were present.

Estimation of production is usually measured by computation between times at wich estimates of population abundance and average fish size are available. In our case, since data were collected in a single time, annual production as fresh weight was estimated indirectly. Considering all the fish present in the population over a year time, based on the effects of size selectivity on population estimates with electrical fishing gear, we have calculated the production using turnover ratio, estimates for fish species (systematic compilation of $\mathrm{P} / \mathrm{B}$ values from literature). Although it involves a rough estimation of production with an associated error, we use this result as an approach to the measure of production to compare our study sites with other rivers inhabited by otters.

As to the aim of this work, which was to evaluate the success of the reintroduction of the Eurasian otter, results show that the study area can sustain a stable density of otter population, similar to other densities present in otter rivers of the Iberian Penninsula (SAAVEDRA, 1995). The carrying capacity depending on fish biomass can be established in 0,4 to 0,9 otters $\cdot \mathrm{km}^{\prime}$ in stretchs with $30-80 \mathrm{~g} \cdot \mathrm{m}^{2}$ and $0,1-0,3$ otters. $\mathrm{km}{ }^{\mathrm{E}}$ when fish biomass is $10-30 \mathrm{~g} \cdot \mathrm{m}^{2}$ (RUIZ-OLMO, 1995). Biomass and fish production present ranges which are able to carry, in the whole area, an otter density of 130- 160 ind. (SAAVEDRA, 1995).

\section{REFERENCES}

RAGENAL, T. 1978. Methods for Assessment of Fish Production in Fresh Waters. Ed. Blackwell Scientific Publications, Oxford. 365 pp.

BOHLIN, T., HAMRIN, S., HEGGBERGET. T.G., RASMUSSEN, G. \& SALTVEIT, S.J. 1989. Electrofishing - Theory and practice with special emphasis on salmonids. Hydrobiologia 173: 9-43.
BOHLIN, T. 1990. Estimation of population parameters using electric fishing: aspects ol the sampling design with emphasis on salmonids in streams. In: Cowx, I.G. (ed.) Developments in Electric Fishing. Fishing News Rooks. Oxford. Chapter 23: 156- 173.

BOHLIN, T. \& COWX, I.G. 1990. Implications of unequal probability of capture by electric fishing on the estimation of population size. In: Cowx, I.G. (ed.) Developments in Electric Fishing. Fishing News Books. Oxford. Chapter 22: 145-155.

BRUSI, D. 1992. Les aigües. In: Pallí, L. \& Brusi, D. (ed.) El medi natural. Diputació de Girona - Universitat de Girona. Girona.

GARCIA-BERTHOU, E. 1993. A computer program for estimating population size by the removal method. Butlletí de la Institució Catalana d 'Histbria Natural, 61: 13- 18.

JøRGENSEN, L.A. 1978. Handbook of Environmental Data and Ecological Parameters. Editorial board: M.B. Friis, J. Henriksen. Norway.

JUNGE, C. O. \& LIBOSVARSKî, J. 1965. Effects of size selectivity on population estimates based on successive removals with electrical fishing gear. Zool. listy. 14, 2: 171-178.

KENNEDY, G.J.A. \& STRANGE C.D. 1981. Efficiency of Electric Fishing for Salmonids in Relation to River Width. Fish. Mgmt 12, No.2: 55-60.

LOBÓN-CERVIÀ, J.. BERNAT, Y. \& RINCON, P.A. 1990. Effects of eel (Anguilla anguilla L.) removals from selected sites of a stream on its subsequent densities. Hidrobiología 206:207-216.

LOBÓN-CERVIÁ, J. \& UTRILLA, C.G. 1992. A simple model to determine stream trout (Salmo trutta L.) densities based on one removal with electrofishing. Fisheries Research. 15: 369-378

MAHON, R. 1980. Accuaracy of catch-effort methods for estimating fish density and biomass in streams. Env. Biol. Fish. Vol. 5, N 4: 343-360.

MANN, R.H.K. \& PENCZAK, T. 1986. Fish production in rivers: A review. Pol. Arch. Hydrobiol. 33: 233-247.

MASON, C.F. \& MACDONALD, S.M. 1986. Otters: ecology and conservation. Cambridge University Press.Cambridge.

MEFFE, G.K. 1984. Eftects of ahiotic disturbance on coexistence of predator-prey fish species. Ecology 65(5): 1525-1534.

PENCZAK, T., LOBÓN-CERCIÁ, J., O’HARA, J. \& JAKUBOWSKI, H. 1986. Production and Food Consumption by Fish Populations in the Pilawa and Dobrzyca Rivers, North Poland. Pol. Arf h. Hydrobiol. 33. 3/4: $345-372$. 
RUIZ-OLMO, J. 1995. La nutria (Lutra lutra) en el NE de la Peninsula Ibérica. Ph. D. Thesis. University of Barcelona.

SAAVEDRA, D. \& SARGATAL, J. 1993. El projecte Ilúdriga: Aiguamolls de l'Empordà i conques de la Muga $i$ del Fluvià. 180 pp. Not published.

SAAVEDRA, D. 1995. Estudi de viabilitat de la reintroduccio de la lludriga al Parc Natural dels Aiguamolls de l'Emporda i conques de Ia Muga i del Fluvià. Informe Projecte Lludriga. $144 \mathrm{pp}$.

SCHNUTE, J. 1983. A new approach to estimating populations by the removal method. Can. J. Fi.sh. Aquar. Sci. $40(12): 2153-2169$.
SEBER, G.A.F. 1982. The estimation of animal abundance and related parameters. Griffin. London .

SIH, A., CROWLEY, P., McPEEK, M., PETRANKA, J. \& STROHMEIRER K. 1985. Predation, competition and prey communities. A review of field experiments. Ann. Rewv. Ecol. Syst. 16: 269-311.

SOSTOA, A. 1990. Les comunitats de peixos continentals. In: Folch, R. (ed.) Histbria Natural dels Països Catalans. Volum 11, Peixos. Enciclopedia Catalana, Barcelona.

ZALEWSKI, M. 1983. The Influence of Fish Community Structure on the Efficiency of Electrofishing. Fish. Mgmt. 14. No.4: 177-186. 